\title{
Sliding Mode Closed-Loop Control of FES: Controlling the Shank Movement
}

\author{
Sašo Jezernik*, Ruben G. V. Wassink, and Thierry Keller
}

\begin{abstract}
Functional electrical stimulation (FES) enables restoration of movement in individuals with spinal cord injury. FES-based devices use electric current pulses to stimulate and excite the intact peripheral nerves. They produce muscle contractions, generate joint torques, and thus, joint movements. Since the underlying neuromuscular-skeletal system is highly nonlinear and time-varying, feedback control is necessary for accurate control of the generated movement. However, classical feedback/closed-loop control algorithms have so far failed to provide satisfactory performance and were not able to guarantee stability of the closed-loop system. Because of this, only open-loop controlled FES devices are in clinical use in spite of their limitations.

The purpose of the reported research was to design a novel closed-loop FES controller that achieves good tracking performance and guarantees closed-loop stability. Such a controller was designed based on a mathematical neuromuscular-skeletal model and is founded on a sliding mode control theory. The controller was used to control shank movement and was tested in computer simulations as well as in actual experiments on healthy and spinal cord injured subjects. It demonstrated good robustness, stability, and tracking performance properties.
\end{abstract}

Index Terms-FES modulation, nonlinear system control, rehabilitation engineering, spinal cord injury.

\section{INTRODUCTION}

$\mathbf{F}$ UNCTIONAL electrical stimulation (FES) enables restoration of functional movement in paralyzed individuals whose lower motoneurons remain intact [1]. In FES, an electric field generated by current pulses delivered via one or more surface or implantable electrodes is used to excite the intact peripheral nerves, which subsequently leads to generation of action potentials that propagate to the muscles and cause them to contract. These muscle contractions produce joint torques, and thus joint movements. The above-mentioned FES principles are used in neuroprosthetic devices to restore, for example, hand grasp, standing or walking. To achieve a desired movement, FES has to be applied in an appropriate way. One way of generating (controlling) the movement is to deliver the stimulation pulses in an open-loop way. The generated movement is, however, not assessed (sensed), and there is no correction applied to the stimulation in case of deviation of

Manuscript received October 2, 2001; revised May 26, 2003. Asterisk indicates corresponding author

*S. Jezernik is with the Swiss Federal Institute of Technology, (ETH Zürich), Automatic Control Laboratory, Physikstrasse 3, 8092 Zürich, Switzerland, and also with the Spinal Cord Injury Center ParaCare, University Hospital Balgrist, Forchstrasse 340, 8008 Zürich, Switzerland (e-mail: jezernik@aut.ee.ethz.ch).

R. G. V. Wassink and T. Keller are with the Swiss Federal Institute of Technology, (ETH Zürich), Automatic Control Laboratory, 8092 Zürich, Switzerland, and also with the Spinal Cord Injury Center ParaCare, University Hospital Balgrist, 8008 Zürich, Switzerland.

Digital Object Identifier 10.1109/TBME.2003.820393 the produced movement from the desired one. The sensing and feedback correction is the main feature of the closed-loop (feedback) control schemes. The latter have several advantages over the open-loop schemes, like better tracking performance and smaller sensitivity to modeling errors, parameter variations, and external disturbances.

Some open and closed-loop FES control strategies that were designed and tested so far were described in [2]. The open-loop FES control performance was however found unsatisfactory for accurate movement control due to existing parameter variations (e.g., muscle fatigue), inherent time-variance, time-delay, and strong nonlinearities present in the neuromuscular-skeletal system (plant to be controlled). The nonlinearities are present in muscle activation relation, muscle dynamics, and skeletal dynamics. Closed-loop control is thus necessary for accurate control of movement [3], [4], and adaptive closed-loop strategies are obviously the most suitable candidates to tackle the FES control problem. Unfortunately, it turned out that closed-loop controller design for FES applications is a rather hard problem [5]. So far classical closed-loop control algorithms have failed to provide satisfactory performance and were not able to guarantee stability, a desired property of the controlled system that was seldom addressed in FES applications. Currently, only open-loop controlled FES devices are in clinical use in spite of their limitations.

Still, recent research efforts dealing with closed-loop control of FES yielded some improvements. For example, in the last decade, neural networks have been incorporated into the control schemes as they are able to learn complex nonlinear mappings (see, e.g., [2], [6], and [7]). Their application yielded better tracking performance, but stability issues remained unresolved due to their black-box structure. Another disadvantage of neural networks is that they are too slow for on-line adaptation and need to be trained off-line. In the case of parameter variation, when FES is applied to different subjects, neural networks often need to be retrained, which is a time consuming procedure. Some other recent FES control approaches included the use of switched/finite-state driven controller schemes for standing up, sitting down, and walking [8]-[10]. These approaches outperformed open-loop control, but still did not achieve very good performance and were unable to guarantee stability. However, they are important as they address different hierarchical control levels and can as well easily integrate the intentions of the patient, which gives the patient increased feelings of safety.

Several groups have recently developed and tested some modern model-based control approaches to control unsupported standing in paraplegia. Unsupported standing would increase the workspace of normally wheelchair dependent paraplegic subjects or could be used as a rehabilitation exercise (important for balance retraining, bone density, cardio-vascular system 
etc.). Explored control approaches included Linear Quadratic Regulator (LQR) design [11]-[13], RST pole-placement [14], and $\mathrm{H}_{\infty}$ robust control theory [15] to design FES controllers that controlled ankle joint torque or ankle joint torque and angular position. However, the problem to control standing is a problem where a static, constant reference angle needs to be maintained (tracked). Future developments and experiments need to be done in order to test the suitability of the developed approaches to address control of FES induced limb movement (with tracking of reference movement trajectories).

The goal of our research was to address the closed-loop FES control design for tracking of limb movement. Our approach was a model-based, nonlinear controller design that guarantees stability and good tracking performance. The design is based on the so-called sliding mode (SM) control theory, which was developed in the 1970s [16]-[18], and was successfully applied, among others, in the robotics field [19], [20]. The SM control is inherently adaptive, and robust to parameter variations. Its application in the FES was suggested for the first time in [21], but was until very recently never realized or directly applied to a real neuromuscular-skeletal system. This was likely due to high complexity of the underlying system. Also, some of the system states are unmeasurable, but would be needed in sliding mode control law calculations. One of the main contributions of our controller design was actually to exploit the special structure of the plant model, which enabled us to avoid the problem of unmeasurable states. The sliding mode FES controller was designed to control shank movement induced by quadriceps stimulation (knee joint angle position control). The controller performance was first examined in extensive computer simulations and later tested on healthy and spinal cord injured (SCI) subjects. The controller design and simulation results were already published in two conference articles [22], [23], but are included again for the sake of completeness.

\section{METHODS}

\section{A. Neuromuscular-Skeletal Human Knee Model}

The human knee model that was used for the controller design consists of three main parts: muscle activation, muscle contraction dynamics, and skeletal dynamics (dynamical equation of motion). The model is a continuous variable model and simulates the effect of electrical stimulation of the knee flexors/extensors on the generated shank movement. The model is two-dimensional (2-D) and the knee joint has one degree of freedom. The model input is the pulsewidth PW (or analogously the current amplitude) of the square-wave current pulses, and the model output is the knee joint kinematics. Chosen model parameters represent a generic (average) person. The parameter values were determined based on experimental data found in literature and by identification/model-validation experiments performed by the group of Dr. Riener, who developed the model. Modeling details were published in [8] and [24].

In our simulations, a sitting position was assumed, with $0^{\circ}$ knee joint angle corresponding to $90^{\circ}$ knee flexion, and $90^{\circ}$ knee joint angle corresponding to full knee extension. In experiments (see below), a slightly different posture and leg position had to be used. Only one muscle model (vastii) was used in the controller design, however, the effect of one or two closed-loop controlled/stimulated muscles (vastii and biceps femoris) was evaluated in computer simulations. The experiments were performed with quadriceps muscle being stimulated by surface electrodes (see below).

The muscle activation block models nerve fiber recruitment with a straight line between the stimulation threshold (PWthr. $=50$ or $100 \mu \mathrm{s}$ ) and the saturation at PWsat. $=$ $500 \mu$ s. In [8] and [24], a sum of two arctan functions and an offset was used to approximate the recruitment curve, but here we have used a piecewise affine approximation instead. The effect of temporal summation and Calcium release and reuptake dynamics was modeled by two identical first order transfer functions (time constants for different muscles had different values, i.e., 0.05 and $0.1 \mathrm{~s}$ for vastii and biceps femoris respectively), and the model also included a time-delay of 25 ms that accounted for action potential propagation along the nerve, the neuromuscular junction and the muscle. The muscle fatigue effect was incorporated into the model by multiplication of a time-varying parameter with the normalized muscle activation. The time variation of this additionally introduced parameter called fitness function was governed by a differential equation that also depended on the stimulation frequency. The stimulation frequency had an effect on muscle fatigue and $\mathrm{Ca}^{2+}$ dynamics (see [8] and [24] for more details).

In the muscle contraction dynamics block, a modified Hilltype muscle model was implemented. The absolute muscle force was thus calculated by a product of muscle activation, muscle force-velocity, and muscle force-length relations. Muscle fiber length and velocity were derived from the knee joint angle and angular velocity, and from the corresponding moment arms.

The skeletal dynamics block calculates the knee joint angle and velocity from a second-order nonlinear differential equation driven by the sum of active and passive knee joint torques. The active knee joint torque is a product of the muscle force and the moment arm, and was scaled to $30 \%-70 \%$ of the average person's torque in order to account for the reduced performance of paralyzed muscles. Passive knee joint torques consisted of linear viscous damping $(\mathrm{d}=0.6)$, nonlinear knee elasticity (modeled by a double exponential function), and gravity terms.

\section{B. The Sliding Mode FES Controller Design}

The neuromuscular-skeletal model was translated into a statespace representation needed for the controller design. The resulting system is

$$
\left[\begin{array}{c}
\dot{x}_{1}=x_{2} \\
\dot{x}_{2}=\frac{m a\left(x_{1}\right) \cdot f i t \cdot x_{3} \cdot g_{1}\left(x_{1}\right) \cdot g_{2}\left(x_{1}, x_{2}\right)+\tau_{g}\left(x_{1}\right)+\tau_{p}\left(x_{1}\right)-d \cdot x_{2}}{I_{\text {tot }}} \\
\dot{x}_{3}=x_{4} \\
\dot{x}_{4}=\frac{1}{T^{2}} \cdot\left(-2 T \cdot x_{4}-x_{3}\right)+\frac{1}{T^{2}} \operatorname{sat}_{\mathrm{rec}}\left[u\left(t-t_{d}\right)\right]
\end{array}\right]
$$

whereby the following notation was used:

- $x_{1}$ : knee joint angle;

- $x_{2}$ : knee angular velocity;

- $x_{3}$ : normalized $\mathrm{Ca}^{2+}$ concentration;

- $x_{4}$ : derivative (rate of change) of the normalized $\mathrm{Ca}^{2+}$ concentration;

and

- ma: moment arm;

- $f i t$ : muscle fitness variable;

- $g_{1}$ : muscle force-length relationship;

- $g_{2}$ : muscle force-velocity relationship; 
- $\tau_{g}$ : gravity torque;

- $\tau_{p}$ : passive elasticity torque;

- $d$ : coefficient of linear viscous damping;

- $T$ : $\mathrm{Ca}^{2+}$ release-reuptake time constant for the vastii muscle;

- $t_{d}$ : time-delay stemming from action potential propagation and delay associated with $\mathrm{Ca}^{2+}$ release from sarcoplasmatic reticulum;

- $s a t_{\mathrm{rec}}$ : static piecewise-linear recruitment curve;

- $I_{\mathrm{TOT}}$ : total moment of inertia;

- $u$ : input to the model (pulse-width or pulse amplitude of the current pulses at stimulation frequency of $20 \mathrm{~Hz}$ ).

The model has a special structure since the derivatives of $x_{1}$ and $x_{2}$ do not depend on $x_{4}$, and the derivatives of $x_{3}$ and $x_{4}$ do not depend on $x_{1}$ and $x_{2}$. Therefore, it can be split into two subsystems as follows:

$$
\begin{aligned}
& \left.\begin{array}{l}
\text { Subsystem 1 } \\
\text { Subsystem 2 } \\
\dot{x}_{2}=f_{2}\left(x_{1} x_{2}, x_{3}\right)
\end{array}\right] . \\
& {\left[\begin{array}{c}
\dot{x}_{3}=f_{3}\left(x_{4}\right) \\
\dot{x}_{4}=f_{4}\left(x_{3} x_{4}, u\right)
\end{array}\right]}
\end{aligned}
$$

The state variable $x_{3}$ can now be regarded as an input to the subsystem 1 and as an output of the subsystem 2 , which is driven by the input $u$. It is important to note that $x_{1}$ and $x_{2}$ can be measured, but $x_{3}$ and $x_{4}$ can not. $x_{2}$ can also be obtained from $x_{1}$ by differentiation. Because the states $x_{3}$ and $x_{4}$ are unmeasurable, we were not able to design a full-state sliding mode controller for the system (1). However, the special structure (2) allowed us to design a sliding mode controller for the first subsystem, and then to invert the subsystem 2 in order to cancel its dynamics and to design an overall FES controller for (1). Approximate inversion of the subsystem 2 was used because of the presence of the time-delay and saturation.

The basic idea behind the sliding mode control [16], [17], [25] is to define a sliding surface (submanifold) in the $\mathfrak{R}^{k}$ vectorspace and to generate a control law (sliding mode control) that will force the system trajectory to reach the sliding surface in a finite time, and that will ensure that the subsequent evolution of the system trajectory will remain on the sliding surface (this latter mode is called a sliding mode). The sliding mode usually describes the error dynamics and forces the error to asymptotically decay to zero.

The sliding surface is defined as $s\left(x(t), x_{\text {reference }}(t)\right)=0$. If we select the control law to guarantee condition $d s / d t \cdot s<0$ [condition (A)] then the sliding mode surface will necessarily be reached (because (A) implies $d s / d t<0$ for $s>0$ and vice versa; i.e. the function $\mathrm{s}$ will decrease if positive and increase if negative). This can be achieved by the following discontinuous control law:

$$
\dot{s}=-k \cdot \operatorname{sgn}(s)
$$

where $k$ is a positive constant.

The control law that will furthermore ensure that $s(t)$ will remain zero [sliding mode, condition (B)] can be calculated from the formula $d s / d t=0$. This control is called the equivalent control, $u_{\text {eq }}$. The two conditions A and B guarantee the reachability and stability of the sliding mode.
As a first step of the FES controller design, an asymptotically stable sliding mode (SM) controller was designed for the first subsystem. The sliding surface was selected as

$$
\begin{aligned}
s\left(x, x_{\mathrm{REF}}\right) & =\left(x_{2, \mathrm{REF}}-x_{2}\right)+\lambda\left(x_{1, \mathrm{REF}}-x_{1}\right) \\
& =e_{2}+\lambda e_{1}=\dot{e}_{1}+\lambda e_{1}
\end{aligned}
$$

where the error $e$ is defined as the difference between the reference and actual angular position $\left(e_{1}\right)$, respectively, velocity $\left(e_{2}\right)$. This choice of the sliding surface was also motivated by the backstepping sliding mode design, see e.g., [25], because if $e_{2}$ equals $-\lambda e_{1}$ then we achieve asymptotic stabilization of the position error $e_{1}$ since $d / d t\left(e_{1}\right)=-\lambda e_{1}$. In our 2-D case, the sliding surface represents a line through the origin of the error (phase) plane with the line having a slope $-\lambda$.

The control law $u_{\mathrm{SM}}=x_{3}$ that combines conditions (A) and (B) for our problem is then given by

$$
\begin{aligned}
x_{3}= & \frac{1}{m a\left(x_{1}\right) \cdot f i t \cdot g_{1}\left(x_{1}\right) \cdot g_{2}\left(x_{1}, x_{2}\right)} \\
& \times\left[-\tau_{g}-\tau_{p}+d \cdot x_{2}+I_{\mathrm{tot}}\right. \\
& \left.\cdot\left(-\lambda x_{2}+k \cdot \operatorname{sgn}(s)+x_{2, \dot{\mathrm{REF}}}+\lambda x_{2, \mathrm{REF}}\right)\right] .
\end{aligned}
$$

It can easily be seen by definition (4) and by inserting (5) into (1) that this discontinuous control law achieves asymptotical stability of knee joint angle and velocity $\left(e_{1} \rightarrow 0\right.$ and $\left.e_{2} \rightarrow 0\right)$, since after the sliding mode is reached $(s(t) \equiv 0)$ it follows that:

$$
\dot{e_{1}}+\lambda e_{1} \equiv 0 \text { and } \quad \dot{e_{2}}+\lambda e_{2} \equiv 0 .
$$

Since it is well known that the discontinuous sliding mode control law introduces chattering (due to high-frequency switching of the controller signal when crossing the sliding surface) [18], [25], we have tested also the continuous sliding mode control law, where the discontinuous term $k \cdot \operatorname{sgn}(s)$ was replaced by a continuous one, $k \cdot s$. The continuous sliding mode control law was reported to be able to reduce chattering [19], [26].

After calculating the sliding mode control law for the first subsystem, we will now deal with the approximate inversion of the subsystem 2 . By neglecting the time-delay $t_{d}$ ( $t_{d}$ was neglected in the current controller design), we obtain the following implicit expression for $u(t)$ :

$$
\text { sat }_{\text {rec }}[u(t)]=T^{2} \ddot{x_{3}}+2 T \dot{x_{3}}+x_{3} .
$$

Since the magnitude of the first term in (7) is smaller than the magnitude of the other two terms (and in order to avoid double differentiation required in (7) and, thus, to reduce the associated sensitivity to noise) the expression in (7) was approximated by its last two terms only, and further low-pass filtered with a first order filter with a time-constant $T_{\mathrm{f}}$. This simplification is justified by the fact that the $\mathrm{L}_{2}$ norm (calculated for a walking trajectory over one movement period) of the second term represents $25 \%$ of the $\mathrm{L}_{2}$ norm of the third term, and the $\mathrm{L}_{2}$ norm of the first term only $3 \%$ of the $\mathrm{L}_{2}$ norm of the third term. The expression (8) with additionally introduced offset-term $\mathrm{C}_{\mathrm{FF}}$ and gains $\mathrm{G}_{\mathrm{SM}}$ and $\mathrm{G}_{\mathrm{TOT}}$ that inverted the effects of the recruitment saturation function was at the end used in all simulations and experiments to calculate the controller output $u(t)$. The term $u_{\mathrm{SM}-\mathrm{SS} 1}$ in (8) equals the expression for $x_{3}$ from (5). The offset term $\mathrm{C}_{\mathrm{FF}}$ 


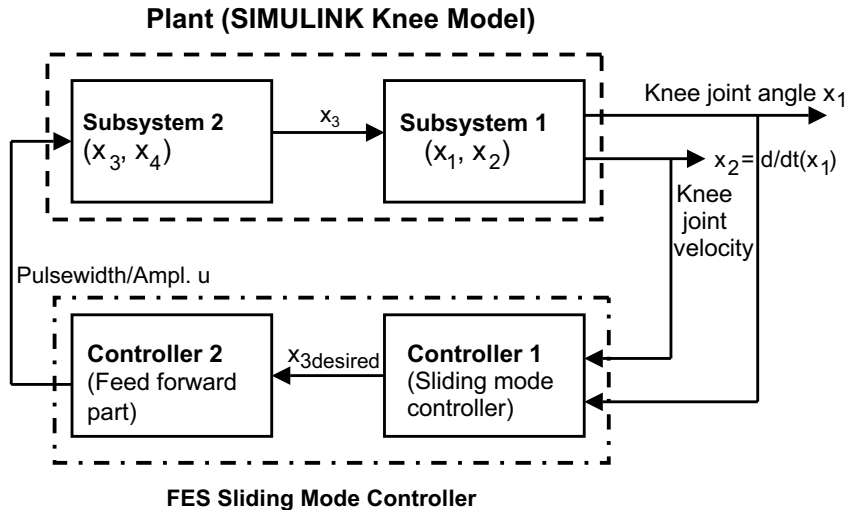

Fig. 1. Block diagram showing the structure of the plant (neuromuscularskeletal model) that can be divided into two subsystems, and the structure of the developed sliding mode FES controller. The input to the plant is either stimulation pulse width or amplitude, and the plant output is the knee joint angle (and knee joint velocity).

was added because of the dead-zone characteristics (stimulation threshold) of the static recruitment curve

$$
u(t)=\left(\frac{\left(2 \cdot T_{t} \cdot s+1\right) \cdot G_{\mathrm{SM}}}{\left(T_{f} \cdot s+1\right)} \cdot u_{\mathrm{SM} \_\mathrm{SS} 1}+C_{\mathrm{FF}}\right) \cdot G_{\mathrm{TOT}} .
$$

The control output (8) was further bounded between the threshold and stimulation saturation levels, and ran through a zero-order hold $(\mathrm{ZOH})$ block that had the sampling time equal to the stimulation frequency (as the stimulation pulses can only be delivered at this frequency). The controller block-diagram is shown in Fig. 1. In this figure, the output of the controller 1 is given by (5), and the output of the controller 2 by (8).

Both variants, the FES controller with discontinuous and continuous sliding mode control law were evaluated in simulations, but in experiments only the continuous variant was used as it expectedly yielded better simulation results (reduced chattering). In this manuscript we, therefore, only include results with the continuous sliding mode controller, whereas simulation results with the discontinuous sliding mode controller were reported in [22].

\section{Computer Simulations}

The simulations were performed in MATLAB/SIMULINK software environment with Runge-Kutta solver and integration time-step of $1 \mathrm{~ms}$. The stimulation frequency was $20 \mathrm{~Hz}$. Tracking performance for step movements as well as ramps and actual knee joint angle trajectories with amplitudes of $60^{\circ}$ and periods of 1,2, 4, and $8 \mathrm{~s}$ were tested.

In the simulations we have first systematically explored the effect of the controller parameters $k$ and $\lambda$ on the tracking performance and stability. The theoretical effect of the controller parameters $k$ and $\lambda$ is, first, how fast the sliding manifold will be reached (proportional to $1 / \mathrm{k}$ ), and, second, how fast the trajectory will slide along the sliding surface toward the origin (proportional to $1 / \lambda$ ). The tracking performance was assessed by calculation of the root-mean-square (RMS) tracking error and by trajectory evolution in the $\mathrm{e}_{1}-\mathrm{e}_{2}$ phase plane. The parameters $T_{t}, T_{f}, C_{\mathrm{FF}}$ and $G_{\mathrm{SM}}$ were tuned to achieve best controller performance (achieved for $T_{t}=0.05, T_{f}=0.01, C_{\mathrm{FF}}=100$, and $\left.G_{\mathrm{SM}}=400\right)$ and $G_{\text {tot }}$ was in simulations set to 1 . It is actually clear from the theory that $T_{t}$ equals the time-constant of the vastii Ca dynamics, and $\mathrm{C}_{\mathrm{FF}} * \mathrm{G}_{\mathrm{TOT}}$ equals the stimulation threshold. GS can be related to the slope of the recruitment saturation function.

Furthermore we have also examined the controller performance in the case of added sensor noise (band-limited noise whose variance could be varied was added to the joint angle measurement), and in the case of deliberately introduced plantmodel mismatch. The latter robustness analysis was performed by varying the estimates of the model terms fit, $I_{\text {tot }}, \tau_{g}$, and $\tau_{p}$ used in the SM control law by $-50 \%,-20 \%, 20 \%$, and $50 \%$, and by comparing the achieved RMS tracking errors with the nominal tracking error. Relative increases/decreases in the tracking errors were calculated and plotted in polar plots (see below).

\section{Actual Experiments}

The experiments were performed using a special experimental set-up consisting of the robotic orthosis Lokomat [27], the Compex Motion stimulator [28], and personal computers running MATLAB package XPC that was used to build and execute the real-time code. The Lokomat is a special robotic orthosis for rehabilitation of locomotion in SCI and stroke patients. It has actuated left and right hip and knee joints (four degrees of freedom). Its movement is conventionally controlled by independent joint proportional derivative position control, whereby the built-in potentiometers are used for the joint angle measurement. The Lokomat was used as a supporting tool in order to provide an easy but reliable fixation of the subject and of the subject's legs. An advantage of using the Lokomat to perform these experiments was also that the knee joint angle could be measured by an built-in Lokomat angular position sensor.

The experimental verification of the controller performance was carried out on six healthy and two SCI subjects. The experiments were performed by placing two surface electrodes connected to the Compex Motion stimulator over the left quadriceps muscle and by strapping the subject into the Lokomat (Fig. 2). The Lokomat itself was controlled so that the right leg position was fixed to standing condition and that the thigh of the left leg was fixed at $40^{\circ}$. The left shank of the subject was allowed to freely swing (with almost no friction), which was achieved by controlling the left Lokomat knee drive to zero force. By this special control, the inertia of the Lokomat and its effects on the subject's lower limb were made negligible. The Lokomat knee angle sensor was used to measure the knee joint angle and to calculate the corresponding knee joint velocity signals that were needed for feedback. The controller output was a biphasic current pulse with an amplitude between zero and, typically, $55-65 \mathrm{~mA}$, a pulse width of $200 \mu \mathrm{s}$, and a stimulation frequency of $20 \mathrm{~Hz}$. The maximal current amplitude sufficient to fully extend the knee was determined experimentally before the actual trials. In the case of one SCI patient, a higher maximal current $(125 \mathrm{~mA})$ was needed due to the presence of muscle atrophy.

We have tested stability and tracking performance for step and ramp movements as well as for a real walking trajectory movement. Tested periods ranged from 1 to $8 \mathrm{~s}$, and the duration of a trial was $20 \mathrm{~s}$ in order to avoid muscle fatigue (between the trials we always had a pause of about $1 \mathrm{~min}$ ). The controller performance was tuned by changing the controller parameters $\lambda, k$, $C_{\mathrm{FF}}, G_{\mathrm{SM}}$, and $G_{\mathrm{TOT}}$. The RMS tracking error was calculated 




(a)

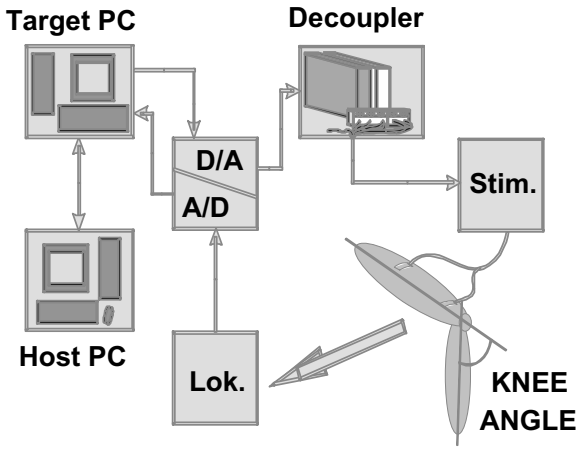

(b)

Fig. 2. (a) Picture of the experimental setup with the legs of the subjects strapped into the robotic orthosis Lokomat. Schematic representation is given in (b). The knee joint angle was measured by the Lokomat angular sensor and fed to the target PC computer that executed the controller task in real-time. This task computed the stimulation current pulse amplitude that was used to modulate the output of the electrical stimulator. Electrical stimulation pulses were delivered by two selfadhesive transcutaneous stimulation electrodes placed over the quadriceps muscle.

(a)
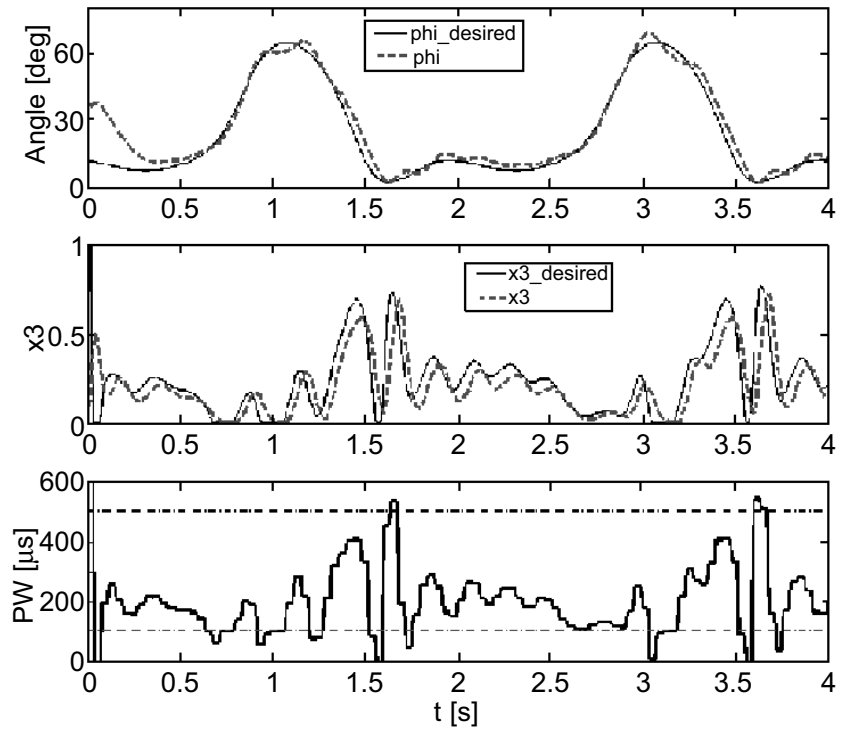

(b)

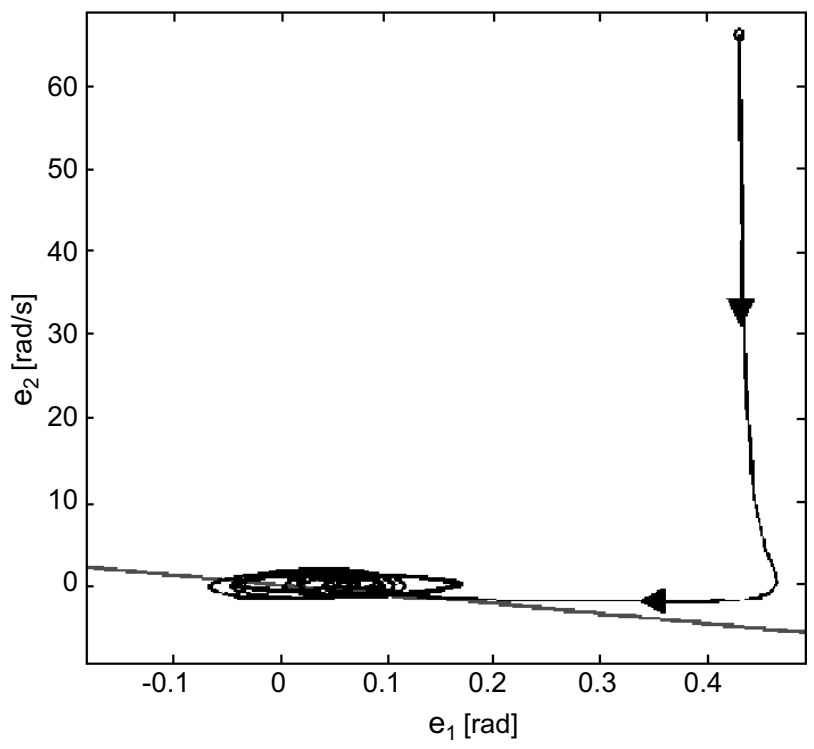

Fig. 3. Computer simulation results when tracking a real knee joint angle walking trajectory. (a) Top graph shows the desired (solid line) and the actual knee joint angle (dashed line). The middle graph shows the corresponding desired (calculated by the sliding mode controller part, solid line) and actual (output of the plant model) state variable $\mathrm{x}_{3}$ (normalized $\mathrm{Ca}^{2+}$ ion concentration). The bottom graph shows the controller output (pulse width). (b) Error phase-plane with the system trajectory calculated during the considered tracking task. The trajectory started at an initial point in the top/right corner of the graph, has then reached the sliding surface (line), and afterwards approached and remained in the vicinity of the zero error point.

for well-tuned controllers and across different trials and subjects. Since only the knee extensors were stimulated and evaluated in the experiments, we report in this manuscript only on the simulation results related to the stimulation of the knee extensor muscle. Simulation results related to the stimulation of the flexor/extensor muscle pair were reported in [22] and [23].

\section{RESULTS}

\section{A. Simulation Results}

The system was stable in all simulations. A typical result of tracking a real walking trajectory with a period of $2 \mathrm{~s}$ and amplitude of $63^{\circ}$ is shown in Fig. 3(a). A very good tracking was achieved (in spite of the fast period), with a RMS error being equal to $2.92^{\circ}$ (approx. $4.6 \%$ of the peak-peak amplitude). The corresponding phase-plot in the $\mathrm{e}_{1}-\mathrm{e}_{2}$ error plane is shown in Fig. 3(b). The trajectory was controlled from the initial position and velocity error point (marked with a circle in the top/right corner) toward the sliding manifold (line), and after reaching the sliding manifold remained in the vicinity of the origin (zero position and velocity errors). The controller parameters also had an effect on the tracking error. This was systematically examined for periodic ramp movements with a period of $2 \mathrm{~s}$ and an amplitude of $60^{\circ}$. The results are summarized in Fig. 4(a). The smallest RMS tracking error was achieved for $k=4$ and $\lambda=20$, and was equal to $2.92^{\circ}$.

Even better tracking results (smoother movement) than shown in Fig. 3(a) were observed if the time-delay $\left(t_{d}=25 \mathrm{~ms}\right)$ 
(a)

\begin{tabular}{|c|c|c|c|}
\hline $\mathrm{k}$ & $\begin{array}{c}\mathrm{e}_{\mathrm{RMS}}[\mathrm{deg}] \\
(\lambda=10)\end{array}$ & $\begin{array}{c}\mathrm{e}_{\mathrm{RMS}}[\mathrm{deg}] \\
(\lambda=20)\end{array}$ & $\begin{array}{c}\mathrm{e}_{\mathrm{RMS}}[\mathrm{deg}] \\
(\lambda=30)\end{array}$ \\
\hline 0.8 & 4.81 & 3.79 & 3.47 \\
\hline 4 & 2.96 & $\mathbf{2 . 9 2}$ & 3.71 \\
\hline 8 & 2.98 & 4.77 & 5.72 \\
\hline 16 & 5.56 & 6.65 & 12.98 \\
\hline
\end{tabular}

(b)

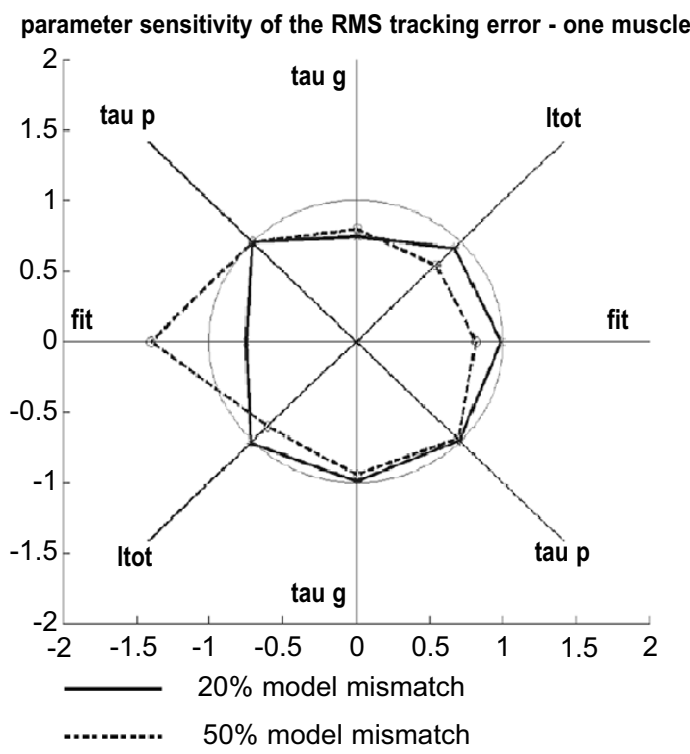

Fig. 4. (a) RMS tracking errors obtained in computer simulations for different values of controller parameters $k$ and $\lambda$ for ramp movement with a period of 2 $\mathrm{s}$ and amplitude of $60^{\circ}$. (b) Relative RMS tracking errors for $k=8$ and $\lambda=20$ with introduced plant model-controller parameter mismatch (relative RMS error=error with mismatch divided by nominal tracking error without mismatch). The relative tracking errors plotted along the top-right four axes were calculated for the overestimated plant model parameter-terms fit, $I_{\mathrm{tot}}, \tau_{g}$, and $\tau_{p}$. Those plotted along the bottom-right four axes for the underestimated plant model parameter-terms. The points calculated for $20 \%$ model mismatch are connected by solid, those for $50 \%$ model mismatch by dashed lines. If the tracking errors with model mismatch have increased compared to the nominal error, the relative tracking error points lay outside the unit circle.

was ignored in the plant model (set to $0 \mathrm{~ms}$ ). In this case the actual knee joint angle was almost identical as the desired angle plotted with solid line in the top panel of the Fig. 3(a). Time-delay, in turn, introduced oscillations of the actual angle around the desired angle trajectory. These oscillations increased with increased $\lambda$ and $k$ (e.g., for $\lambda>20$ and $\mathrm{k}>10$ ) and were the reason for increased tracking errors shown in the last row/column of Fig. 4(a).

The oscillations also increased when we added the measurement noise to the knee joint angle measurement. However, the system remained stable and the controller performance was still good (but worse than in absence of the time-delay and/or measurement noise).

The robustness of the controller performance to modeling errors (model/controller parameter mismatch) was systematically examined by introducing a modeling mismatch of $20 \%$ and $50 \%$ into control law terms, and by calculating the RMS tracking errors for different mismatches while tracking a ramp movement with a period of $2 \mathrm{~s}$ and amplitude of $60^{\circ}$. The controller parameters were set to $k=4$ and 8 , and $\lambda=20$ (nominal error for the case of exact model/controller term match was $4.77^{\circ}$ for $k=8$ ). It could be observed that the robustness increased for higher $\mathrm{k}(k=8)$ compared to $k=4$. For $k=8$ the relative increases/decreases in the RMS tracking error were plotted in a polar plot [Fig. 4(b)]. If the tracking error for specific parameter term mismatch was greater than the nominal error, the resulting relative error point layed outside the unit circle, and if the tracking error was smaller, the resulting relative error point was inside the unit circle area. As we can see in Fig. 4(b), the tracking error remained the same or even decreased in all cases but for a $50 \%$ underestimated fitness term, fit, where the error increased by approximately 1.5 times $\left(1.5 * 4.77=7.15^{\circ}\right)$. Fig. $4(\mathrm{~b})$, thus, demonstrates the inherent robustness of the developed controller, which was further confirmed in the experiments.

\section{B. Experimental Results}

The best controller performance was achieved by selecting $\lambda$ and $k$ to have values between 5 and 10 (typically both values equaled 8). Higher $\lambda$ and $k$ values led to more oscillatory tracking. The values for $\mathrm{C}_{\mathrm{FF}}$ ranged from 250 to $400, \mathrm{G}_{\mathrm{SM}}$ from 450 to 950 (once 1800 and once 2000), and $\mathrm{G}_{\mathrm{TOT}}$ always equaled 0.01 to translate the controller output value of $500(\mu \mathrm{s})$ to $5 \mathrm{~V}$, which corresponded to maximal stimulation current. $\mathrm{T}_{\mathrm{t}}$ was equal to 0.05 and $T_{f}$ to 0.01 .

The values for the above parameters were selected independently for each subject such that the response of the controlled system was as good as possible. Full examination (determination of optimal parameter values) was not possible due to limited experimental time. In many trials there was no need to change the controller parameters that were used earlier on a different subject.

Still, the sliding mode FES controller achieved good tracking results and remained stable in all experiments. Instability was never observed. There was no qualitative or quantitative difference in performance when controlling the movement of the shank in the case of healthy and SCI subjects (except in the case of a patient with severe muscular atrophy).

The achieved RMS tracking errors when controlling movements with $20^{\circ}$ amplitude are listed in Table I, in the left part of the table. Relative tracking errors calculated by dividing the RMS errors by the peak-to-peak (P-P) amplitude of the corresponding movement are shown in Table I in the right part (for all movements with amplitudes ranging from $20^{\circ}$ to $30^{\circ}$ ). The RMS tracking errors typically amounted to $2.5^{\circ}$ to $5.5^{\circ}$. The mean relative errors equaled $21.6 \%, 17.5 \%$, and $23.8 \%$ for step, ramp, and knee walking trajectory movements, respectively. The tracking errors in one patient were larger than in the other experiments due to the presence of muscle atrophy. How- 
TABLE I

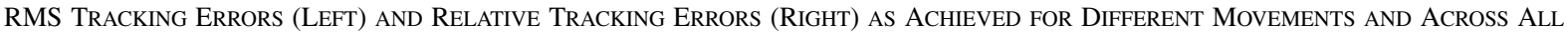
EXPERIMENTS. $\mathrm{N}=$ NUMBER OF TRIALS

\begin{tabular}{|c|c|c|c|c|c|c|c|}
\hline \multirow[t]{2}{*}{ Movement } & \multicolumn{3}{|c|}{ RMS-Errors } & \multirow[t]{2}{*}{ Movement } & \multicolumn{3}{|c|}{$\begin{array}{c}\text { Relative Errors } \\
\text { (RMS-Error/P-P Ampl.) }\end{array}$} \\
\hline & Mean & St.Dev. & $\mathbf{N}$ & & Mean & St.Dev. & $\mathbf{N}$ \\
\hline Step & 4.33 & 1.09 & 13 & Step & $21.6 \%$ & $5.4 \%$ & 13 \\
\hline Ramps & 3.43 & 0.48 & 9 & Ramps & $17.5 \%$ & $2.4 \%$ & 23 \\
\hline $\begin{array}{l}\text { Knee } \\
\text { Traj. }\end{array}$ & 4.76 & 0.85 & 16 & $\begin{array}{l}\text { Knee } \\
\text { Traj. }\end{array}$ & $23.8 \%$ & $4.5 \%$ & 23 \\
\hline
\end{tabular}

(a)
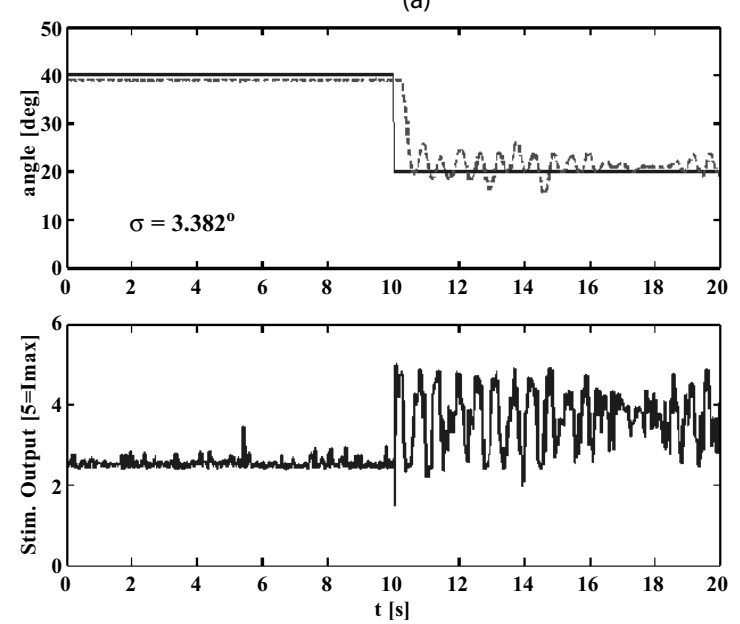

(b)
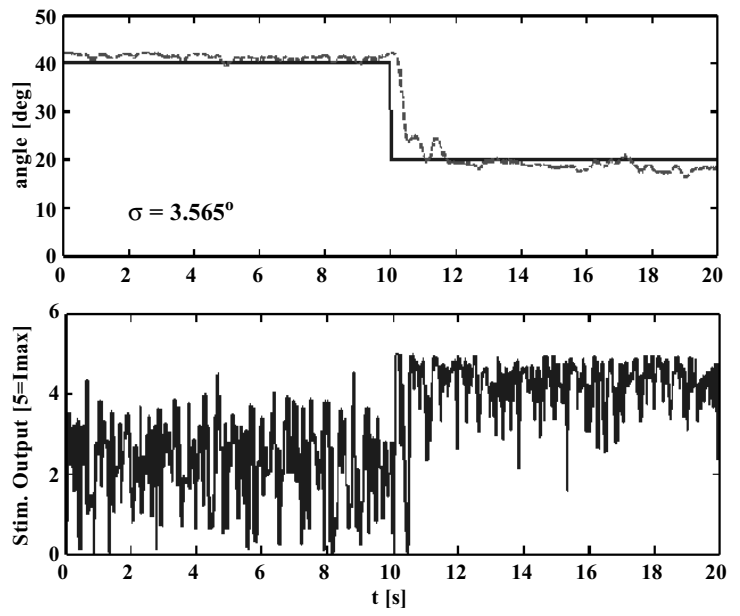

Fig. 5. Experimental data obtained during a controlled step movement in (a) a healthy and (b) a SCI individual. The bottom graphs show the stimulation current pulse amplitude (controller output), and the top graphs the desired (solid line) and the actual knee joint angle (dashed line).
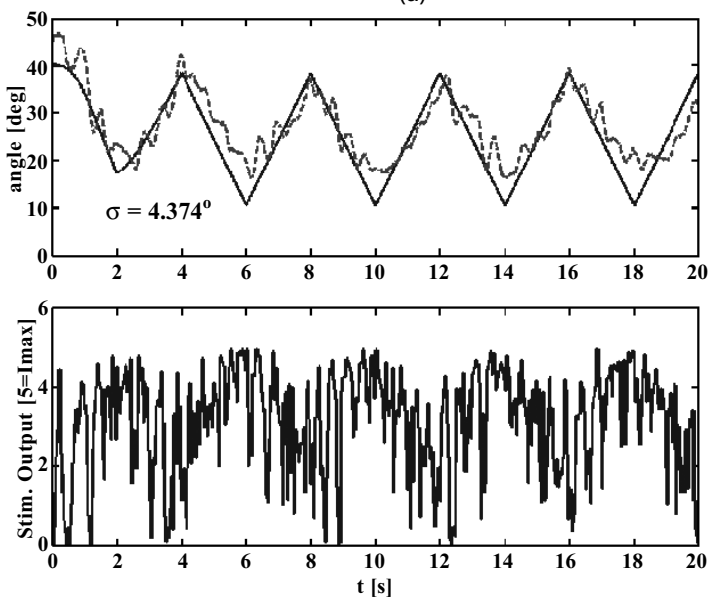

(b)
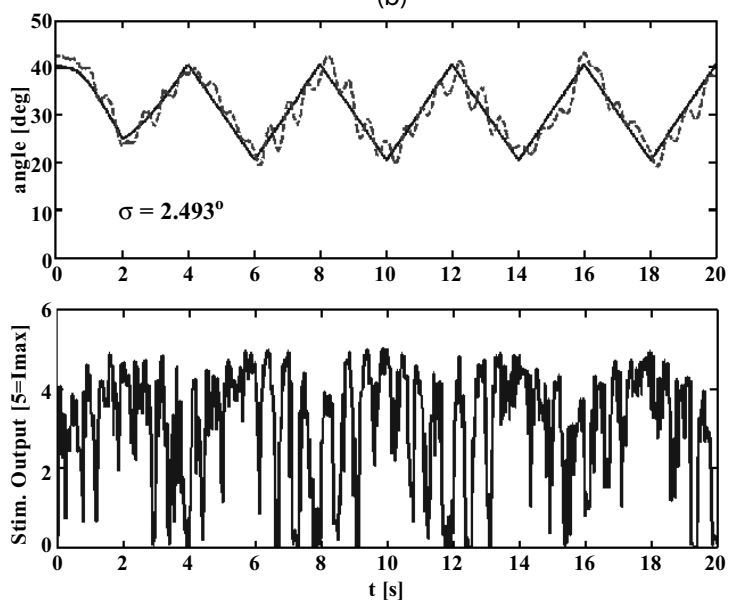

Fig. 6. Experimental data obtained during controlled ramp movements in (a) a healthy and (b) a SCI individual. The ramp movements had a period of $4 \mathrm{~s}$. The bottom graphs show the stimulation current pulse amplitude (controller output), and the top graphs the desired (solid line) and the actual knee joint angle (dashed line).

ever, the tracking errors with the other patient were in most cases smaller than with healthy subjects.

Fig. 5(a) shows an example of controlled step movement (exp8, healthy subject, RMS error $3.38^{\circ}$ ). The top panel shows the desired (solid line) and measured knee joint angle (dashed line), and the bottom panel the corresponding controller output (amplitude of the stimulation current). Smaller angles correspond to knee extension and larger angles to knee flexion.
After the step, small oscillations around the desired angular value can be observed. These oscillations typically increased with increased gain or increased $\lambda$ and $k$, and could be reduced by decreasing these parameters. Fig. 5(b) shows the controlled step movement in case of a paraplegic patient (exp11, RMS error $3.56^{\circ}$ ). Tracking of ramp movements with a period of $4 \mathrm{~s}$ is shown in Fig. 6(a) (exp16, healthy subject, RMS error 4.37 ${ }^{\circ}$ ) and 6(b) (exp11, patient, RMS error $2.49^{\circ}$ ). Two examples of 
(a)
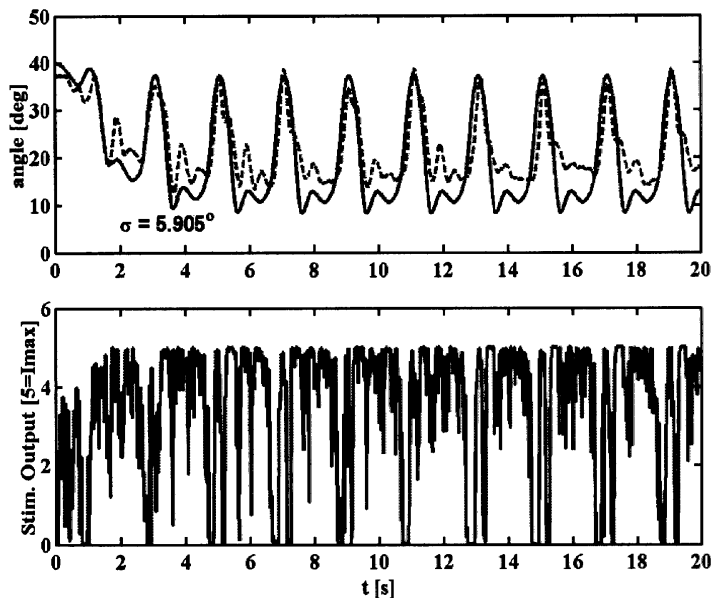

(b)
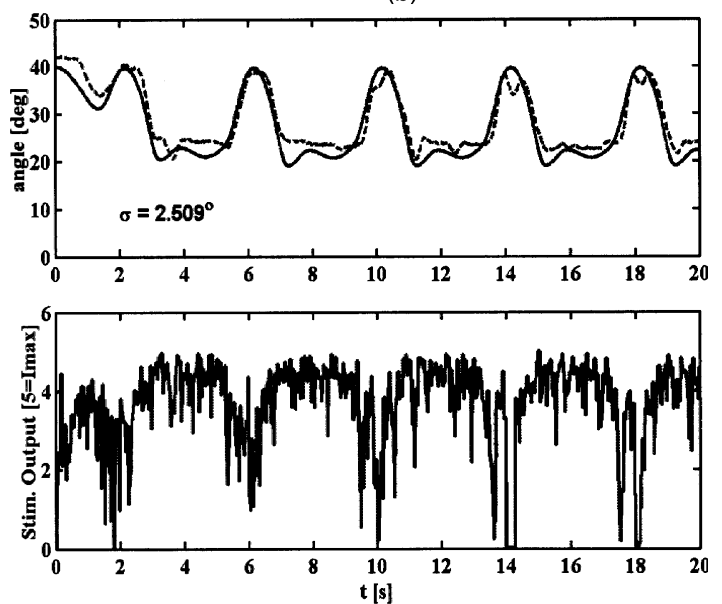

Fig. 7. As in Figs. 5 and 6, but the tracked movement in the case of (a) the healthy subject was an actual knee joint walking trajectory with a period of 2 s, whereas in the case of (b) the SCI individual, the tracked movement was an actual knee joint walking trajectory with a period of $4 \mathrm{~s}$.

(a)
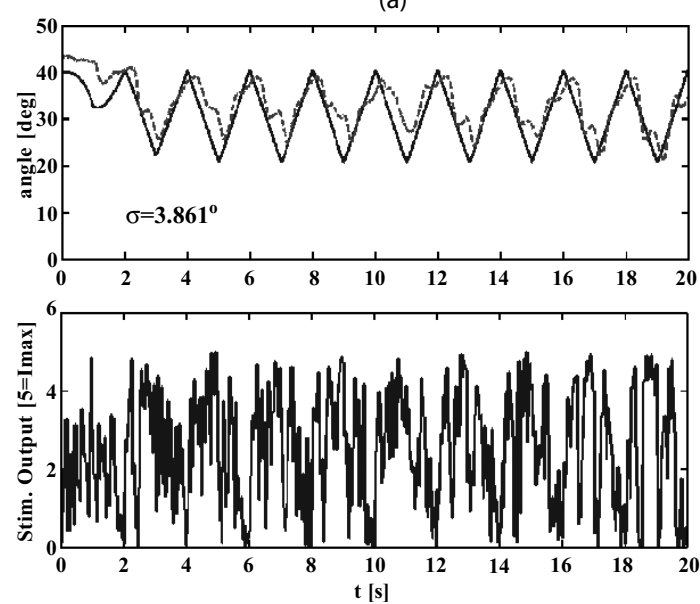

(b)
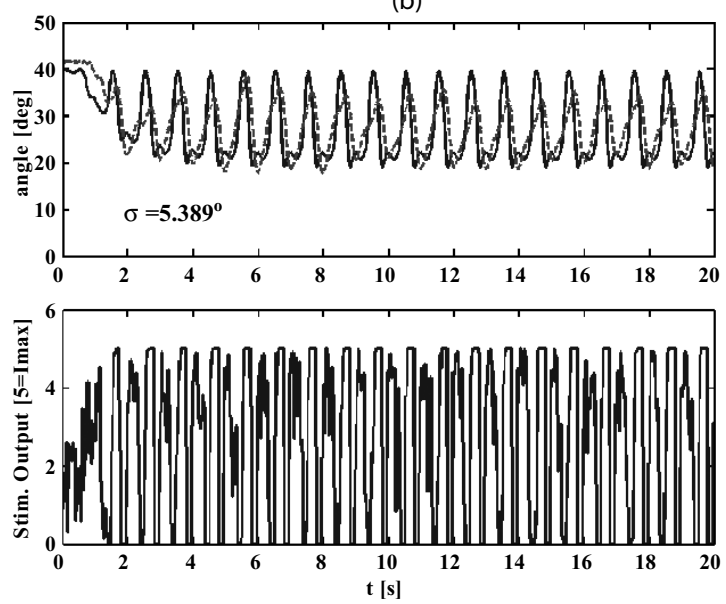

Fig. 8. Additional tracking results measured with healthy subjects. (a) Tracking of a ramp movement with a period of $2 \mathrm{~s}$, and (b) tracking of an actual knee joint walking trajectory with a period of $1 \mathrm{~s}$.

tracking a real knee joint angle walking trajectory are shown in Fig. 7(a) and 7(b) (A: exp15, healthy subject, RMS error 5.90 , period $2 \mathrm{~s}$; B: exp11, patient, RMS error $2.50^{\circ}$, period $4 \mathrm{~s}$ ). In Fig. 7(a) we can see that the controller output saturated at $5 \mathrm{~V}$ (maximal current) during periods of maximal knee extension, and that the desired extension levels could not be reached.

Fig. 8 demonstrates tracking of a faster movement recorded in exp10 (healthy subject). Fig. 8(a) shows tracking of a ramp with period of $2 \mathrm{~s}$ (RMS error $3.86^{\circ}$ ), and Fig. 8(b) tracking of the knee walking trajectory with a period of $1 \mathrm{~s}$ (RMS error $\left.5.38^{\circ}\right)$. In the latter case we can see that the controller achieved the desired maximal extension, however the system was too slow to reach the desired flexion (downward movement) fast enough. The controller had to work in a kind of bang-bang regime in order to try to track such a fast movement.

\section{DISCUSSION}

To the knowledge of the authors the developed FES controller represents the first FES controller that is purely modelbased and guarantees stability in a system and control theoretical manner. Instability was never observed neither in simulations nor in experiments. The controller performance in exper- iments was good, but worse than in simulations. This can be attributed to quite high levels of sensor noise and unavoidable model-plant mismatches. More precise identification of model parameters in case of each specific subject might lead to better performance, but is very time consuming and should, thus, be avoided. The sliding mode design was chosen just to automatically provide robustness for plant- model mismatch, and to avoid the need of precise model parameter identification and tuning. Moreover, most of the controller parameters (e.g., fit, $\left.g_{1}, g_{2}, T, d\right)$ are difficult or impossible to be estimated individually. We believe that we have succeeded in designing a robust FES controller, as the controller worked well during experiments with just average parameters and was only tuned by changing a small set of parameters (for example, the inertia of the plant used in control law calculation was never changed during experiments even though the mass of the subjects varied from subject to subject). Many times, there was even no need to modify the controller parameters that were used in an earlier experiment on another subject.

More promise to improve the performance of the presented controller lies in the following approaches: 1. reduction of the sensor noise, since simulations have shown that sensor noise 
greatly impairs the performance, and 2., employment of additional techniques to compensate for the time-delay $t_{d}$, since the simulations have again shown that the time-delay is greatly responsible for worse performance versus simulations that neglected the presence of the time-delay in the plant. Some approaches to address 2 could include the theory of Model Predictive Control or the theory of Smith Predictor. These could easily be designed to deal with the time-delay present in the linear subsystem 2.

Another possibility to further improve the controller performance could be to increase the stimulation frequency from $20 \mathrm{~Hz}$ to higher frequencies. This would increase the controller bandwidth. On the other hand, the muscles fatigue faster when stimulated with higher frequencies. Therefore, the optimal stimulation frequency must take into account both these two aspects. The effect of stimulation frequency on the tracking performance was recently tested in another study on three subjects, and the overall results were published in [29]. The main result of this study was that the optimal stimulation frequency with the respect to muscle fatigue and tracking performance is in the range of $30-40 \mathrm{~Hz}$. This is higher than the stimulation frequency that was used in the present study.

Interesting would also be to try to develop a sliding mode controller based on a simplified model. However, further model simplifications could easily deteriorate the performance. Initial work with such a development was very recently reported in [30], but the controller was tested only on one subject with only one special form of the reference trajectory that had a period of $20 \mathrm{~s}$. This direction, thus, necessitates further research engagement.

During the fast movements (period $<=1.5 \mathrm{~s}$ ) we have observed that the leg did not perform the desired knee flexion fast enough. During a fast knee flexion, the knee extensors were not stimulated, and the knee was flexed just due to the gravity torque. Knee flexion could be accelerated if knee flexors were stimulated to generate additional knee flexion torque. This was so far only shown in simulations, and was reported in [22] and [23]. Experiments with two-channel stimulation of the knee flexor/extensor muscle pair will, thus, be performed next. Knee flexor stimulation would also be needed to generate knee joint angles greater than $40^{\circ}$ in the Lokomat experiments, where the hip had to be flexed for about $40^{\circ}$. The special Lokomat set-up with maximal hip flexion of $40^{\circ}$ was responsible for reduced amplitudes of experimentally tested movements $\left(20^{\circ}-30^{\circ}\right.$ versus $60^{\circ}$ in simulations, where a sitting position with $90^{\circ}$ hip flexion was assumed).

The adaptation of the control law for the case of concurrent control of a flexor/extensor muscle pair, and especially for the case of the sliding mode FES controller augmented to control two or three joints will need further developments. The plan is to address these in the near future. Accurate closed-loop control of movements produced by several joints might be important to improve the function of the existing neural prostheses. Multijoint controller would especially be important for closed-loop control of standing up and sitting down, and for closed-loop control of arm and hand movement (reaching, grasping). Hand dexterity is well known to necessitate a precise motor control. To achieve these goals, new, modified sliding mode controllers will need to be developed. However, these controllers are expected to evolve as extensions of the FES controller presented in this manuscript.

\section{CONCLUSION}

A novel closed-loop FES controller for shank movement was designed and tested in computer simulations and actual experiments on healthy and SCI subjects. The controller achieved good tracking performance and the closed-loop system was always stable. A good tracking performance in the case of SCI subjects precluded absence of severe muscular atrophy. If the muscular atrophy limits the muscle force to such an extent that the maximal muscle force that can be generated with electrical stimulation is not sufficient to achieve a proper range of motion, the controller of course cannot compensate for this incapacity of the actuator.

Computer simulations were very helpful to examine the effects of variation of different controller parameters on the controller performance. They have furthermore demonstrated the expected robustness in presence of modeling errors. This robustness allowed a very fast controller tuning in case of different subjects, since the model parameters in the control law were not needed to be exactly known and, thus, there was no need to identify them for each subject separately.

The achieved results hold great promise to improve and augment the developed controller, and to actually employ it in implantable or external neural prostheses that necessitate accurate control of FES generated movements.

\section{ACKNOWLEDGMENT}

The experiments were performed at the Spinal Cord Injuries Center Paracare, University Hospital Balgrist, Zurich, Switzerland. The authors would like to thank Dr. R. Riener for kindly providing them the neuromuscular-skeletal model.

\section{REFERENCES}

[1] A. Kralj and T. Bajd, Functional Electrical Stimulation: Standing and Walking After Spinal Cord Injury. Boca Raton, FL: CRC, 1989.

[2] P. E. Crago, N. Lan, P. H. Veltink, J. J. Abbas, and C. Kantor, "New control strategies for neuroprosthetic systmes," J. Rehab. Res. Dev., vol. 33, no. 2, pp. 158-172, Apr. 1996.

[3] H. J. Chizeck, P. E. Crago, and L. S. Kofman, "Robust closed-loop control of isometric muscle force using pulsewidth modulation," IEEE Trans. Biomed. Eng., vol. 35, pp. 510-517, July 1988.

[4] H. J. Chizeck, N. Lan, L. S. Palmieri, and P. E. Crago, "Feedback control of electrically stimulated muscle using simultaneous pulse width and stimulus period modulation," IEEE Trans. Biomed. Eng., vol. 38, pp. 1224-1234, Dec. 1991.

[5] M. Ferrarin, F. Palazzo, R. Riener, and J. Quintern, "Model-based control of FES-induced single joint movements," IEEE Trans. Neural Syst. Rehab. Eng., vol. 9, pp. 245-257, Sept. 2001.

[6] N. Lan, H.-Q. Feng, and P. E. Crago, "Neural network generation of muscle stimulation patterns for control of arm movements," IEEE Trans. Rehab. Eng., vol. 2, pp. 213-224, Dec 1994.

[7] G.-C. Chang, J.-J. Luh, G.-D. Liao, J.-S. Lai, C.-K. Cheng, B.-L. Kuo, and T.-S. Kuo, "Neuro-control system for the knee joint position control with quadriceps stimulation," IEEE Trans. Rehab. Eng., vol. 5, pp. 2-11, Mar. 1997.

[8] R. Riener and T. Fuhr, "Patient-driven control of FES supported standing-up: a simulation study," IEEE Trans. Rehab. Eng., vol. 6, pp. 113-124, June 1998.

[9] R. Riener, M. Ferrarin, E. E. Pavan, and C. A. Frigo, "Patient-driven control of FES-supported standing up and sitting down: Experimental results," IEEE Trans. Rehab. Eng., vol. 8, pp. 523-529, Dec. 2000.

[10] T. Fuhr and G. Schmidt, "A supervisory control approach to restore locomotion in paraplegics with WALK!," in Proc. 7th Ann. IFESS Conf., Ljubljana, Slovenia, June 2002, pp. 310-312. 
[11] K. J. Hunt, M. Munih, and N. Donaldson, "Feedback control of unsupported standing in paraplegia. Part I: optimal control approach," IEEE Trans. Rehab. Eng., vol. 5, pp. 331-340, 1997.

[12] Z. Matjacic and T. Bajd, "Arm-free paraplegic standing. Parts I and II," IEEE Trans. Rehab. Eng., vol. 6, pp. 125-138, pp. 139-150, June 1998.

[13] M. Mihelj and M. Munih, "Minimum effort optimal control of ankle joints for unsupported standing in paraplegia," in Proc. 7th Ann. IFESS Conference, Ljubljana, Slovenia, June 2002, pp. 295-297.

[14] K. J. Hunt, H. Gollee, R. P. Jaime, and N. de N. Donaldson, "Design of feedback controllers for paraplegic standing," Inst. Elect. Eng Proc.Control Theory Appl., vol. 148, no. 2, pp. 97-108, 2001.

[15] K. J. Hunt, R. P. Jaime, and H. Gollee, "Robust control of electricallystimulated muscle using polynomial $\mathrm{H}_{\infty}$ design," Contr. Eng. Practice, vol. 9, pp. 313-328, 2001.

[16] V. U. Utkin, Sliding Modes in Control and Optimization. Berlin, Germany: Springer-Verlag, 1992.

[17] J.-J. E. Slotine, "Sliding controller design for nonlinear systems," Int. J. Contr., vol. 40, no. 2, pp. 421-434, 1984.

[18] J.-J. E. Slotine and J. A. Coetsee, "Adaptive sliding controller synthesis for nonlinear systems," Int. J. Contr., vol. 43, no. 6, pp. 1631-1651, 1986.

[19] A. Šabanovic, K. Jezernik, and K. Wada, "Chattering-free sliding modes in robotic manipulators control," Robotica, pt. 1, vol. 14, pp. 17-29, 1995.

[20] K. Jezernik, M. Rodic, R. Šafaric, and B. Curk, "Neural network sliding mode robot control," Robotica, vol. 15, pp. 23-30, 1997.

[21] W. K. Durfee, "Control of standing and gait using electrical stimulation: influence of muscle model complexity on control strategy," Prog. Brain Res., vol. 97, pp. 369-381, 1993.

[22] S. Jezernik, P. Inderbitzin, T. Keller, and R. Riener, "Sliding mode control of functional electrical stimulation for knee joint angle tracking," in Proc. 7th Vienna Int. Workshop Functional Electrical Stimulation, Vienna, Austria, Sept. 2001, pp. 160-163.

[23] — , "A novel sliding mode controller for functional electrical stimulation," in Proc. 15th IFAC World Congr. Automatic Control, vol. Q, Barcelona, Spain, July 2002, pp. 199-203.

[24] R. Riener, J. Quintern, and G. Schmidt, "Biomechanical model of the human knee evaluated by neuromuscular stimulation," J. Biomech., vol. 29, no. 9, pp. 1157-1167, 1996.

[25] H. K. Khalil, Nonlinear Systems, 2nd ed. Englewood Cliffs, NJ: Prentice-Hall, 1996.

[26] B. Curk, K. Jezernik, J. Harnik, and A. Sabanovic, "Chattering reduction in VSC systems of robotic manipulators," in Proc. 13th IFAC World Congr. Automatic Control, San Francisco, CA, 1996, pp. 503-508.

[27] G. Colombo, M. Joerg, R. Schreier, and V. Dietz, "Treadmill training of paraplegic patients with a robotic orthosis," J. Rehab. Res. Dev., vol. 37, pp. 693-700, 2000.

[28] T. Keller, M. R. Popovic, I. P. Pappas, and P. Y. Muller, "Transcutaneous functional electrical stimulator 'Compex Motion'," Artif. Organs, vol. 26, pp. 219-223, 2002.

[29] S. Jezernik, "The effect of stimulation frequency on the closed-loop control of quadriceps stimulation: Experimental results," in Proc. 5th IFAC Symp. Modeling and Control in Biomedical Systems, Melbourne, Australia, Aug. 2003, pp. 151-155.

[30] T. Schauer, W. Holderbaum, and K. J. Hunt, "Sliding-mode control of knee-joint angle: Experimental results," in Proc. 7th Annu. IFESS Conf., Ljubljana, Slovenia, June 2002, pp. 316-318.

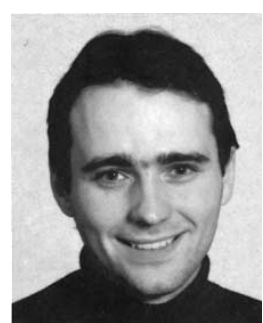

Sašo Jezernik was born in Maribor, Slovenia, in 1971. He received the Dipl.lng. degree in electrical engineering (M.Sc.E.E.) with specialization in control theory and process automation from the Technical University of Graz, Graz, Austria, in 1996, and the Ph.D. degree in biomedical engineering from Aalborg University, Aalborg, Denmark, in 1999.

During study for the Ph.D. degree, he spent seven months as a Visiting Research Scholar at the Applied Neural Control Laboratory, Case Western Reserve University, Cleveland, OH. Since October 1999, he has been a Postdoctoral Assistant at the Automatic Control Laboratory, Swiss Federal Institute of Technology, Zurich, Switzerland. His research interests are in the fields of automatic control, signal processing, and neural prostheses. His past work included whole nerve cuff recording, electrical stimulation, neurophysiology of the lower urinary tract, and closed-loop control of FES systems. Lately, he has been working on the development of gait-pattern adaptation algorithms for the robotic orthosis Lokomat, on advanced control algorithms to control neural prostheses and Lokomat, and on biologically inspired neural network modeling and control.

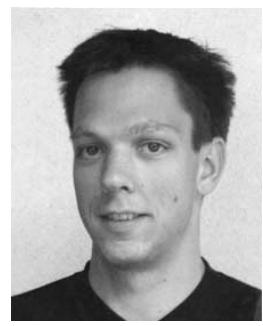

Ruben G. V. Wassink was born in Arnhem, The Netherlands, in 1978. He is currently finishing the M.S. degree studies in electrical engineering with specialization in biomedical engineering at the University of Twente, Twente, The Netherlands.

During his studies the practical training term was done at the Automatic Control Laboratory, Swiss Federal Institute of Technology and University Hospital Balgist (ParaCare) in Zurich, Switzerland. Currently he is working on his master thesis entitled "Automated task classification of human tasks using Hidden Markov Modeling" at Roessingh Research \& Development in Enschede, The Netherlands.

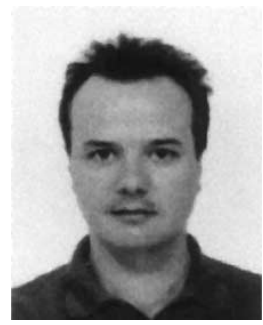

Thierry Keller was born in Bern, Switzerland, in 1968. He received the Dr. SC. techn. and Dipl. Ing. degrees in electrical engineering (M.Sc.E.E.) from the Swiss Federal Institute of Technology Zurich (ETHZ), Zurich, Switzerland, in 2001 and 1995, respectively.

$\mathrm{He}$ is currently working as a Visiting Research Scholar in the Department of Physical Therapy and Human Movement Science, Northwestern University, Chicago, IL. and the Sensory Motor Performance Program, Rehabilitation Institute of Chicago. In addition, he is leading the Functional Electrical Stimulation Group at ETHZ and the Paraplegic Center of University Hospital Balgrist, Zurich, where he held the position of Research Engineer and Research Associate between 1995 and 2002. He developed various neuroprostheses that are used to improve walking and grasping functions in spinal cord injured and stroke subjects. His research interests are in the fields of rehabilitation engineering, neural prostheses, signal processing, and human-machine interaction.

In 2002, Mr. Keller was awarded with a Swiss National Science Foundation (SNF) fellowship for advanced researchers and in 1997 he received the SNF SPP Biotechnology:Technology Transfer Award-1st place. 